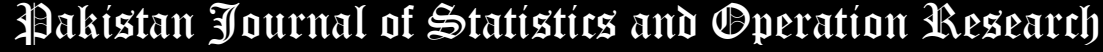

\section{A novel iterative method to solve a linear fractional transportation problem}

\author{
Sema Akin Bas ${ }^{1 *}$, Hale Gonce Kocken², Beyza Ahlatcioglu Ozkok ${ }^{3}$ \\ * Corresponding Author
}

1. Department of Mathematics, Yildiz Technical University, Istanbul, Turkey, akins@yildiz.edu.tr

2. Department of Mathematical Engineering, Yildiz Technical University, Istanbul, Turkey, hgonce@yildiz.edu.tr

3. Department of Business Administration, Yildiz Technical University, Istanbul, Turkey, bahlat@yildiz.edu.tr

\begin{abstract}
The linear fractional transportation problem (LFTP) is widely encountered as a particular type of transportation problem (TP) in real-life. In this paper, a novel algorithm, based on the traditional definition of continuity, is presented to solve the LFTP. An iterative constraint is constructed by combining the objective function of the LFTP and the supply-demand condition since the fractional objective function is continuous at every point of the feasible region. By this constraint obtained, LFTP is converted into an iterative linear programming (LP) problem to reach the optimum solution. In this study, the case of asymptotic solution for LFTP is discussed for the first time in the literature. The numerical examples are performed for the linear and asymptotic cases to illustrate the method, and the approach proposed is compared with the other existing methods to demonstrate the efficiency of the algorithm. Also, an application had environmentalist objective is solved by proposed mathematical method using the software general algebraic modeling system (GAMS) with data set of the real case. Finally, some computational results from tests performed on randomly generated large-scale transportation problems are provided.
\end{abstract}

Key Words: Linear Fractional Programming, Fractional Transportation Problem, Iterative Method, Mixed Constraints, Optimization.

Mathematical Subject Classification: 90C05, 90C32, 90B06

\section{Introduction}

Linear fractional programming (LFP) is encountered more in recent years because it is more realistic in expressing real-life problems such as resource allocation, transportation, location selection, production, stochastic processes, finance, applied linear algebra, game theory, etc. The LFP problem (such as maximization of profitability rates, minimization of risk rates), a particular case of a non-linear programming problem, has the ratio of the linear numerator and denominator as the objective function. To solve an LFP problem, it can be converted into a traditional LP problem by using the method of Charnes and Cooper transformation (Charnes and Cooper, 1962), or it can be adopted the updated objective function method of Bitran and Novaes (1973). Schaible (1981) presented to survey applications as well as solution methods in linear, quadratic, and concave-convex fractional programming. Tantawy (2008) proposed an iterative process based on the conjugate gradient projection method to solve LFP problems with inequality constraints. Ozkok (2020) proposed an iterative method to solve all LFP problems. A detailed bibliography of LFP can be found in (Ozkok, 2020). 
The TP includes decisions having great importance in logistics and supply chain management in terms of reducing costs and providing the best service. The TP with a fractional objective is a widely used LFP problem having ratios such as profit/cost, income/capital, profit/labor, total actual transportation cost/total standard transportation cost, and risk assets/capital, etc. Firstly, Swarup (1966) dealt with a fractional transportation problem (FTP) and supposed that the denominator is always positive. Moanta (2007) studied to obtain an optimum solution with the simplex method for the three-dimensional TP having the objective function as the ratio of two positive linear functions. Sheikhi et al. (2018) proposed a new method allowing decision-makers to evaluate economic activities and make satisfying managerial decisions by finding a set of efficient solutions to bi-objective FTPs.

The FTPs and their solution methods are among the main topics of operations research. Adapting each new method developed for LFP is becoming increasingly important to solve FTP. Guzel et al. (2012) transformed FTP with interval coefficient to a classical TP by expanding the order $1^{\text {st }}$ Taylor polynomial series with multi variables in their study. Raina et al. (2018) handled the FTP with some discount cost during the shipment time and tested the optimum solution by using the Karush-Kuhn-Tucker optimality algorithm.

Mostly iterative methods attract the attention of companies and decision-makers today in order to reach the acceptable solution faster. Gupta et al. (1993) studied a paradox in LFTPs with 'mixed constraints'. Moreover, they established sufficient conditions for the existence of a paradoxical solution and obtained a paradoxical range of flow. Sivri et al. (2011) developed a new algorithm looking like the Vogel approximation method for TP to find an initial point for LFTP. Also, they constituted the optimality conditions. Gupta and Arora (2017) presented a paradoxical method to solve capacitated FTP which has the sum of two fractional functions. Pradhan and Biswal (2015) studied on two algorithms to obtain an initial feasible point of an LTFP, and presented a methodology to get the optimum solution. Gupta and Arora developed a paradoxical method to solve FTP which has the sum of two fractional functions.

Some researchers have tried to overcome the real-life uncertainties by handling FTP with multi-objective and using fuzzy solution approaches for the stated problem solution. Cetin and Tiryaki (2014) ensured a fuzzy approach obtaining a compromise Pareto-optimal solution for a multi-objective LFTP by constructing Generalized Dinkelbach's Algorithm. Taking into consideration on Zadeh's extension, Liu (2016) handled two-level mathematical programs to compute the fuzzy objective value of the problem with fuzzy cost coefficients and fuzzy right-hand side parameters. Sadia et al. (2016) modeled the problem determining the optimum transportation schedule as multi-objective capacitated FTP with mixed constraints. They presented a fuzzy programming approach having linear, exponential, and hyperbolic forms of membership functions. Also, they obtained a compromise solution by using lexicographic goal programming with minimum distance techniques. Safi and Ghasemi (2017) studied on three approaches for solving the LFTP with uncertain parameters. Javaid et al. (2017) modeled transportation problem with multiple fractional objectives with uncertainty. They reached the compromise solution of the problem by using fuzzy goal programming approach. Gupta et al. (2018) studied on the multi-objective capacitated transportation problem with linear and fractional objective functions. They formulated the parameters of problem with fuzzy numbers and were transformed into deterministic form through a ranking function approach. Anukokila et al. (2019) investigated a goal 
programming approach to solve multi-objective FTP by using the parameters $(\gamma, \delta)$ from the point of interval-valued fuzzy numbers.

In the literature, there are no many studies on iterative approaches for the solution of LFTP, a particular type of LFP recently. Besides, some methods, mostly based on matrix operations, give cause for losing their computational effectiveness by reason of increasing process as the problem size raises. On the other hand, our approach overcomes successfully this shortcoming in terms of computation since it is an iterative procedure based on LP. Moreover, variable transformation occurs in many papers in the literature, but our algorithm does not need it.

In this study, we have extended the new iterative algorithm proposed by Ozkok (2020) for an LFP to the LFTP. The fractional objective of the LFTP and the convergence condition are combined to generate an iterative constraint. We create an iterative LP problem with constraint generated to get the optimum solution of the LFTP having a feasible bounded region. We note that our algorithm can solve the LFTP with mixed constraints since our iterative method depends on only the fractional objective function. Moreover, the case of asymptotic solution for LFTP is handled for the first time as far as it is examined in the literature. The computational steps of the proposed approach are carried out more quickly than other algebraic methods. The solution procedure is demonstrated the two numerical examples in literature and a practical case study is presented.

The rest of the paper is arranged as follows. The definition of the LFTP and some preliminaries are mentioned in Section 2. In the next section, we describe the solution methodology. We introduce our algorithm and present its flow chart in Section 4. There are experiments in order to illustrate our algorithm in Section 5. The last section emphasizes our conclusions.

\section{Problem definition and preliminaries}

LFTP is a problem optimizing the rate of profitability expressed as cost/ profit, time/profit or the cost/amount of materials to be transported under supply and demand constraints. The mathematical model of a general LFTP can be stated as follows:

$$
\begin{aligned}
& \min Z(\mathbf{x})=\frac{\mathrm{P}(\mathbf{x})}{Q(\mathbf{x})}=\frac{\sum_{\mathrm{i}=1}^{m} \sum_{\mathrm{j}=1}^{n} \mathrm{p}_{\mathrm{ij}} \mathrm{x}_{\mathrm{ij}}+\mathrm{p}_{0}}{\sum_{\mathrm{i}=1}^{m} \sum_{\mathrm{j}=1}^{n} \mathrm{q}_{\mathrm{ij}} \mathrm{x}_{\mathrm{ij}}+\mathrm{q}_{0}} \\
& \qquad \begin{array}{l}
\sum_{\mathrm{j}=1}^{\mathrm{n}} \mathrm{x}_{\mathrm{ij}} \leq,=, \geq \mathrm{a}_{\mathrm{i}}, \mathrm{i}=1, \ldots, m \\
\sum_{\mathrm{i}=1}^{\mathrm{m}} \mathrm{x}_{\mathrm{ij}} \leq,=, \geq \mathrm{b}_{\mathrm{j}}, \mathrm{j}=1, \ldots, n \\
\mathrm{x}_{\mathrm{ij}} \geq 0, \forall \mathrm{i}, \mathrm{j}
\end{array}
\end{aligned}
$$


where $\mathrm{P}=\left[\mathrm{p}_{\mathrm{ij}}\right]_{m \times n}$ and $Q=\left[\mathrm{q}_{\mathrm{ij}}\right]_{m \times n}$ are matrices for linear functions of numerator and denominator, respectively, $\mathrm{p}_{0}$ and $\mathrm{q}_{0}$ are constant, $\mathrm{a}_{\mathrm{i}}\left(\mathrm{a}_{\mathrm{i}}>0, \forall \mathrm{i}\right)$ is the amount of supply from the $i^{\text {th }}$ source, $\mathrm{b}_{\mathrm{j}}\left(\mathrm{b}_{\mathrm{j}}>0, \forall \mathrm{j}\right)$ is the amount of demand from the $j^{\text {th }}$ region, and $\mathbf{x}=\left(\mathrm{x}_{11}, \mathrm{x}_{12}, \ldots, \mathrm{x}_{m n}\right)$ is decision variable which refers to product amount transported from $i^{\text {th }}$ supply source to $j^{\text {th }}$ demand region. We assume that $P(\mathbf{x})>0$ (Since it is an objective for the TP, it will always be positive.), $Q(\mathbf{x})>0, \forall \mathbf{x}=\left(\mathrm{x}_{i j}\right) \in S$, where the feasible region $S$ is a convex and non-empty feasible set defined by constraints (1b)-(1d), and $Z: S \subset \mathbf{R}^{m n} \rightarrow \mathbf{R}(S) \subset \mathbf{R}$. Moreover, we suppose that

$$
\sum_{\mathrm{i}=1}^{m} \mathrm{a}_{\mathrm{i}} \geq \sum_{\mathrm{j}=1}^{n} \mathrm{~b}_{\mathrm{j}}
$$

which is a necessary and sufficient condition for the existence of a feasible solution to the problem (1a)-(1d). Because the total supply is not less than the total demand.

Theorem 1: LFTP is solvable if and only if the above inequality (2) holds.

Proof: It can be found on page 247 of (Bajalinov, 2003).

Remark 1: With the assumption $Q(\mathbf{x})>0$, the objective function $Z(\mathbf{x})$ is continuous on $\mathbf{R}$, and its domain $S$. It means that $Z(\mathbf{x})$ is continuous on $\forall \mathbf{x}^{k} \in S$. Note that $Z\left(\mathbf{x}^{k}\right)=Z^{k}$.

The continuity can be stated in terms of traditional neighborhoods as follows: $\forall \varepsilon \in R^{+}$, if there exists $\delta>0$ such that $\forall \mathbf{x} \in B\left(\mathbf{x}^{k}, \delta\right)$ and $Z \in B\left(Z^{k}, \varepsilon\right)$ where $Z\left(\mathbf{x}^{k}\right)=Z^{k}$, then $Z(\mathbf{x})$ is continuous at the given $\mathbf{x}^{k} \in \mathbf{R}^{m n}$.

\section{Our methodology}

Considering the algorithm in (Ozkok, 2020), the convergence ensures for every $(Z, \mathbf{x}) \in B\left(Z^{k}, \varepsilon\right) \times B\left(\mathbf{x}^{k}, \delta\right)$ and the convergence condition for the LFTP (1)

$$
Z \mathbf{x}=Z^{k} \mathbf{x}+\mathbf{x}^{k} Z-Z^{k} \mathbf{x}^{k}
$$

is obtained. From the fractional objective function (1a) of LFTP, we get

$$
\sum_{\mathrm{i}=1}^{m} \sum_{\mathrm{j}=1}^{n} \mathrm{q}_{\mathrm{ij}} \mathrm{Zx}_{\mathrm{ij}}+\mathrm{Zq}_{\mathrm{o}}=\sum_{\mathrm{i}=1}^{m} \sum_{\mathrm{j}=1}^{n} \mathrm{p}_{\mathrm{ij}} \mathrm{x}_{\mathrm{ij}}+\mathrm{p}_{0}
$$

Combining the convergence condition (3) and the other expression of the fractional objective function (4), we have:

$$
\sum_{\mathrm{i}=1}^{m} \sum_{\mathrm{j}=1}^{n} \mathrm{q}_{\mathrm{ij}}\left(\mathrm{Z}^{k} \mathrm{x}_{\mathrm{ij}}+\mathrm{Zx}_{\mathrm{ij}}{ }^{k}-\mathrm{Z}^{k} \mathrm{x}_{\mathrm{ij}}{ }^{k}\right)+\mathrm{Zq}_{0}-\sum_{\mathrm{i}=1}^{m} \sum_{\mathrm{j}=1}^{n} \mathrm{p}_{\mathrm{ij}} \mathrm{x}_{\mathrm{ij}}-\mathrm{p}_{0}=0
$$

Following the algebraic operations, 


$$
\mathrm{Z}^{k}\left[\sum_{\mathrm{i}=1}^{m} \sum_{\mathrm{j}=1}^{n} \mathrm{q}_{\mathrm{ij}} \mathrm{x}_{\mathrm{ij}}+\mathrm{q}_{0}\right]-\mathrm{Z}^{k}\left[\sum_{\mathrm{i}=1}^{m} \sum_{\mathrm{j}=1}^{n} \mathrm{q}_{\mathrm{ij}} \mathrm{x}_{\mathrm{ij}}{ }^{k}+\mathrm{q}_{0}\right]+\mathrm{Z}\left[\sum_{\mathrm{i}=1}^{m} \sum_{\mathrm{j}=1}^{n} \mathrm{q}_{\mathrm{ij}} \mathrm{x}_{\mathrm{ij}}{ }^{k}+\mathrm{q}_{0}\right]-\sum_{\mathrm{i}=1}^{m} \sum_{\mathrm{j}=1}^{n} \mathrm{p}_{\mathrm{ij}} \mathrm{x}_{\mathrm{ij}}-\mathrm{p}_{0}=0
$$

and noting that $\mathrm{Z}^{k}=\mathrm{Z}\left(\mathrm{x}^{k}\right)=\frac{\sum_{\mathrm{i}=1}^{m} \sum_{\mathrm{j}=1}^{n} \mathrm{p}_{\mathrm{ij}} \mathrm{x}_{\mathrm{ij}}{ }^{k}+\mathrm{p}_{0}}{\sum_{\mathrm{i}=1}^{m} \sum_{\mathrm{j}=1}^{n} \mathrm{q}_{\mathrm{ij}} \mathrm{x}_{\mathrm{ij}}{ }^{k}+\mathrm{q}_{0}}$,

$$
\mathrm{Z}^{k}\left[\sum_{\mathrm{i}=1}^{m} \sum_{\mathrm{j}=1}^{n} \mathrm{q}_{\mathrm{ij}} \mathrm{x}_{\mathrm{ij}}+\mathrm{q}_{0}\right]-\sum_{\mathrm{i}=1}^{m} \sum_{\mathrm{j}=1}^{n} \mathrm{p}_{\mathrm{ij}} \mathrm{x}_{\mathrm{ij}}{ }^{k}-\mathrm{p}_{0}+\mathrm{Z}\left[\sum_{\mathrm{i}=1}^{m} \sum_{\mathrm{j}=1}^{n} \mathrm{q}_{\mathrm{ij}} \mathrm{x}_{\mathrm{ij}}{ }^{k}+\mathrm{q}_{0}\right]-\sum_{\mathrm{i}=1}^{m} \sum_{\mathrm{j}=1}^{n} \mathrm{p}_{\mathrm{ij}} \mathrm{x}_{\mathrm{ij}}-\mathrm{p}_{0}=0
$$

or

$$
\sum_{\mathrm{i}=1}^{m} \sum_{\mathrm{j}=1}^{n}\left(\mathrm{Z}^{k} \mathrm{q}_{\mathrm{ij}}-\mathrm{p}_{\mathrm{ij}}\right) \mathbf{x}+\left[\sum_{\mathrm{i}=1}^{m} \sum_{\mathrm{j}=1}^{n} \mathrm{q}_{\mathrm{ij}} \mathbf{x}^{k}+\mathrm{q}_{0}\right] \mathrm{Z}=\sum_{\mathrm{i}=1}^{m} \sum_{\mathrm{j}=1}^{n} \mathrm{p}_{\mathrm{ij}} \mathbf{x}^{k}+\mathrm{p}_{0}-\mathrm{Z}^{k} \mathrm{q}_{0}+\mathrm{p}_{0}
$$

or

$$
\sum_{\mathrm{i}=1}^{m} \sum_{\mathrm{j}=1}^{n}\left(Z^{k} q_{\mathrm{ij}}-\mathrm{p}_{\mathrm{ij}}\right) \mathbf{x}+\left[\sum_{\mathrm{i}=1}^{m} \sum_{\mathrm{j}=1}^{n} q_{\mathrm{ij}} \mathbf{x}^{k}+q_{0}\right] Z=\sum_{\mathrm{i}=1}^{m} \sum_{\mathrm{j}=1}^{n} Z^{k} q_{\mathrm{ij}} \mathbf{x}^{k}+\mathrm{p}_{0}
$$

is obtained. $Z$ and $\bar{Z}$ are used to explain the objective function for the LFTP and the LP problem, respectively. The following iterative LP problem denominated $\operatorname{LFTP}-\operatorname{LP}_{(k)}$ is constructed to solve the LFTP (1):

$\min \bar{Z}$

$$
\begin{array}{ll}
\text { s.t. } & \sum_{\mathrm{i}=1}^{m} \sum_{\mathrm{j}=1}^{n}\left(Z^{k} q_{\mathrm{ij}}-\mathrm{p}_{\mathrm{ij}}\right) \mathbf{x}+\left[\sum_{\mathrm{i}=1}^{m} \sum_{\mathrm{j}=1}^{n} q_{\mathrm{ij}} \mathbf{x}^{k}+q_{0}\right] \bar{Z}=\sum_{\mathrm{i}=1}^{m} \sum_{\mathrm{j}=1}^{n} Z^{k} q_{\mathrm{ij}} \mathbf{x}^{k}+\mathrm{p}_{0} \\
\mathbf{x} \in S &
\end{array}
$$

Here, the superscript $k \in\{0,1,2, \ldots\}$ points out the iteration counter and (6.2) denotes our iterative constraint. Our algorithm starts with an initial point $\left(\mathbf{x}^{0}, Z^{0}\right)$, that is $k=0$. By using the initial solution, a second feasible solution $\left(\mathbf{x}^{1}, Z^{1}\right)$ is obtained with the help of the sub-problem (6). Then, another feasible solution $\left(\mathbf{x}^{2}, Z^{2}\right)$ is determined by using the previous feasible solution $\left(\mathbf{x}^{1}, Z^{1}\right)$, and so on. As a rule, if $\left(\mathbf{x}^{k}, Z^{k}\right)$ is a feasible solution achieved at iteration $k$, then the solution procedure goes on with a new feasible solution $\left(\mathbf{x}^{k+1}, Z^{k+1}\right)$ at iteration $k+1$.

Proposition 1: Let $\mathbf{x}^{0} \in S$ be an initial point, and $\mathbf{x}^{1}, \mathbf{x}^{2}, \ldots, \mathbf{x}^{k}, \mathbf{x}^{k+1}, \ldots$ be the successive optimum solutions of (6) $\forall k \in N$ (the iteration counter). Then, the fractional objective function values come up with a decreasing sequence for $\mathbf{x}^{1}, \mathbf{x}^{2}, \ldots, \mathbf{x}^{k}, \mathbf{x}^{k+1}, \ldots$, that is $Z^{0} \geq Z^{1} \geq \ldots \geq Z^{k} \geq Z^{k+1} \geq \ldots$ until reaching the optimum solution of (1). 
Proof: It can be proved easily by keeping up with (Ozkok, 2020).

Proposition 2: The gradient vectors of the fractional objective function $Z$ and the linear objective function $\bar{Z}$ are equal at every point $\mathbf{x}^{k} \in S$.

Proof: Please see (Ozkok, 2020).

Result: Suppose that $\overline{\mathbf{x}^{*}}$ refers to the optimum solution of the problem. The gradient direction specifies the direction of the decrease of a function. As a result of this, a decrease in the linear objective function $\bar{Z}\left(\mathbf{x}^{k}\right) \geq \bar{Z}\left(\overline{\mathbf{x}^{*}}\right)$ implies a decrease in the fractional objective function $Z\left(\mathbf{x}^{k}\right) \geq Z\left(\overline{\mathbf{x}^{*}}\right)$.

\subsection{Determining an initial solution}

Firstly, the initial point needs to be determined to start the algorithm. Choosing an initial solution $\left(\mathbf{x}^{0}, Z^{0}\right)$, the only condition to be considered is that the fractional objective function must not be undefined at that point. In this study, the initial feasible point is chosen by solving the following LP problem:

$$
1^{0} \cdot \min _{\mathbf{x} \in S} 0
$$

Since we are solving LFTP, it is possible to obtain the initial feasible solution by using The Northwest Corner Method (or upper left-hand corner), Maximum Profit (or Minimum Cost) Method, and Vogel's Method which are adapted to LFTP by Bajalinov (2003).

\subsection{Determining the stopping criterion}

$1^{0}$. Considering Proposition 1, a decreasing sequence consists of the fractional objective thanks to the optimum solutions of the auxiliary iterative LP problem (6). If the LFTP (1) has an optimum solution $\mathbf{x}^{*}$ resulted in a finite value $Z^{*}$, then the corresponding sequence will be bounded below. The new solution obtained in each iteration comes close to $Z^{*}$. In this way, if the objective functions are achieved the same value in two successive iterations, then $Z^{k+1^{*}}=Z^{k^{*}}$ can be identified as the stopping criterion.

$2^{0}$. When the least the decision variable $x_{i j}$ approaches infinity under supply-demand constraints, if the objective function of LFTP converges to a finite limit value, then this situation is called the asymptotic case. In this case, the minimum value of the fractional objective function will be gotten at the position vector line of the feasible region (Ozkok, 2020). That is, $\inf _{x \in S} Z(\mathbf{x})=Z^{k}$.

$3^{0}$. When $\mathbf{x}$ goes to infinity, if the fractional objective function approaches infinity, then the LFTP is unbounded.

\section{Execution of the proposed algorithm}

Considering the previous statements, our algorithm is as follows: 
Step 1: Choose the initial solution $\left(\mathbf{x}^{0}, Z^{0}\right)$ for the LFTP (1) and $M$ which is a very big number, set $k=0$.

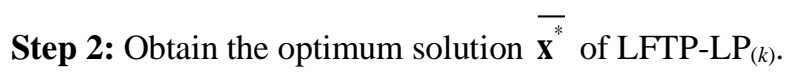

Step 3: Find the product value of $1 \cdot \overline{\mathbf{x}^{*}}$.

Step $3_{\mathrm{i}}$ : If $\mathbf{1} \cdot \overline{\mathbf{x}^{*}}=M$, then take $\mathbf{x}^{k+1}=\frac{1}{M} \overline{\mathbf{x}^{*}}$ and $Z^{k+1}=\frac{\sum_{\mathrm{i}=1}^{m} \sum_{\mathrm{j}=1}^{n} \mathrm{p}_{\mathrm{ij}} \mathbf{x}^{k+1}}{\sum_{\mathrm{i}=1}^{m} \sum_{\mathrm{j}=1}^{n} \mathrm{q}_{\mathrm{ij}} \mathbf{x}^{k+1}}$.

Step $3_{\mathrm{ii}}$ : If $\mathbf{1} \cdot \overline{\mathbf{x}^{*}} \neq M$, then take $\mathbf{x}^{k+1}=\overline{\mathbf{x}^{*}}$ and $Z^{k+1}=\frac{P\left(\mathbf{x}^{k+1}\right)}{Q\left(\mathbf{x}^{k+1}\right)}$.

Step 4: Take into consideration $Z^{k+1}$.

Step $4_{\mathbf{i}}$ : If $Z^{k+1} \rightarrow \infty$, then the LFTP (1) is unbounded. STOP.

Step $4_{\mathrm{ii}}$ : If $Z^{k+1}>Z^{k}$, then the asymptotic solution occurs as the infimum of all alternative solutions: $\inf _{x \in S} Z(\mathbf{x})=Z^{k}$. STOP.

Step $4_{\text {iii }}$ : If $Z^{k+1}=Z^{k}$, then $\mathbf{x}^{*}=\mathbf{x}^{k+1}$ is the optimum solution and $Z^{*}=Z^{k+1}$ is the optimum value of LFTP. STOP.

Step $\mathbf{4}_{\text {iv }}$ : If $Z^{k+1} \neq Z^{k}$, then put $k=k+1$, and return to Step 2 .

The flow chart of the proposed method is illustrated in Figure 1. The algorithm is presented with the direction of the minimum objective. On the other hand, we notify that the procedure can be updated to the direction of the maximum objective with respect to the structure of the LFTP thanks to the flexibility of the algorithm. 


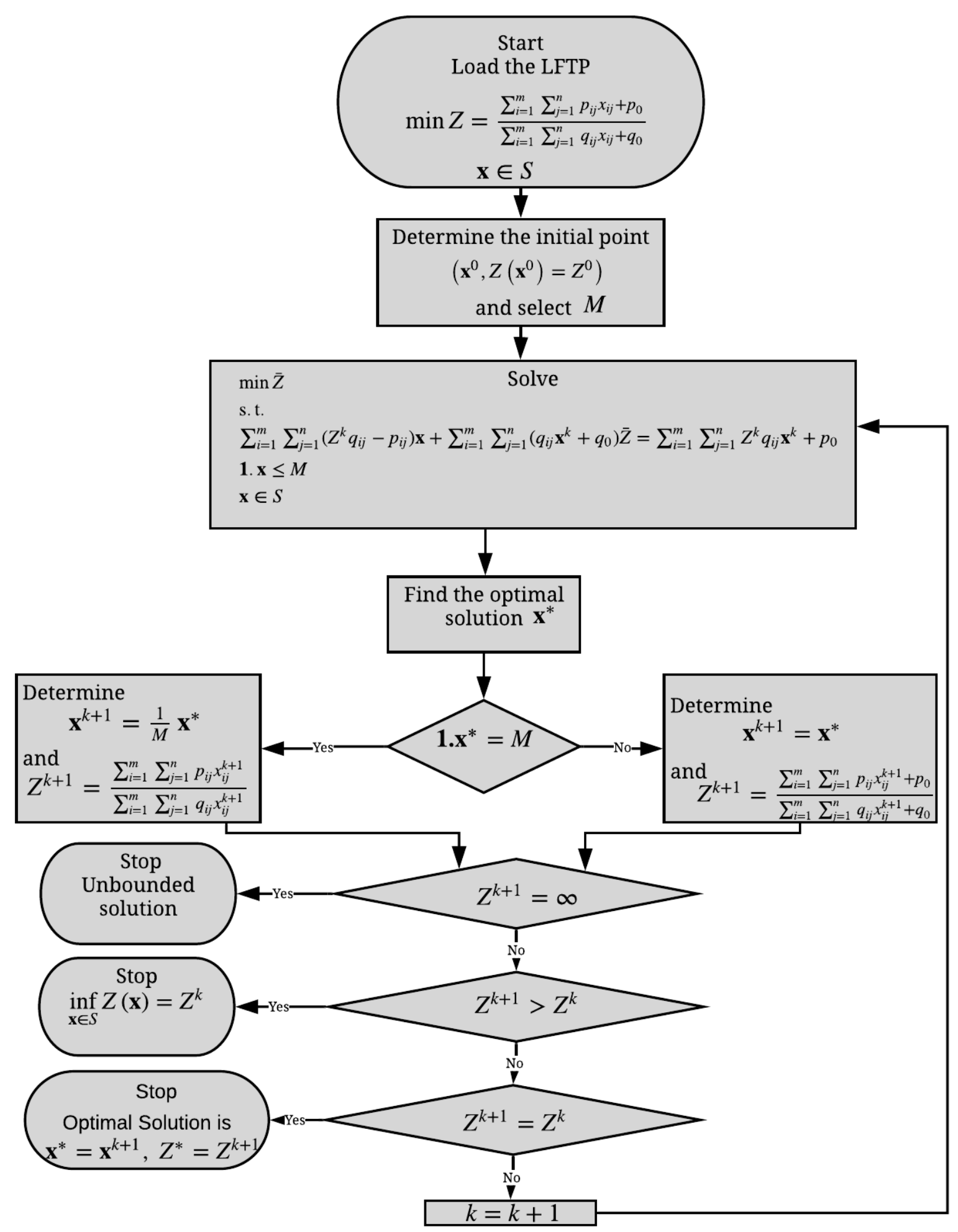

Figure 1: Flow chart of our algorithm for LFTP having minimization objective. 


\section{Numerical illustrations}

In this section, the new introduced solution method is made easier to understand with examples in literature for different cases and a real-life problem. Randomly generated LFTP problems are also carried out to demonstrate the performance of the presented algorithm.

\subsection{Examples}

Example 1 (Bajalinov, 2003) (Optimum solution case): The following LFTP is considered

$$
\begin{array}{ll}
\max & Z(\mathbf{x})=\frac{10 x_{11}+14 x_{12}+8 x_{13}+12 x_{14}+8 x_{21}+12 x_{22}+14 x_{23}+8 x_{24}+9 x_{31}+6 x_{32}+15 x_{33}+9 x_{34}+100}{15 x_{11}+12 x_{12}+16 x_{13}+8 x_{14}+10 x_{21}+6 x_{22}+13 x_{23}+12 x_{24}+13 x_{31}+15 x_{32}+12 x_{33}+10 x_{34}+120} \\
\text { s.t. } \quad & x_{11}+x_{12}+x_{13}+x_{14} \leq 150 \\
& x_{21}+x_{22}+x_{23}+x_{24} \leq 250 \\
& x_{31}+x_{32}+x_{33}+x_{34} \leq 200 \\
& x_{11}+x_{21}+x_{31} \geq 150 \\
& x_{12}+x_{22}+x_{32} \geq 250 \\
& x_{13}+x_{23}+x_{33} \geq 50 \\
& x_{14}+x_{24}+x_{34} \geq 150 \\
& \mathbf{x} \geq 0
\end{array}
$$

Let $S$ denotes the feasible region.

Step 1: The initial solution $\mathbf{x}^{0}=(0,0,0,150,0,200,50,0,150,50,0,0)$ is obtained, and the fractional objective $Z^{0}=\frac{6650}{5870} \cong 1.1329$ is achieved. We also choose $M=10^{10}$.

Step 2: Using $\mathbf{x}^{0}$ and $Z^{0}$, the problem $\operatorname{LFTP}-\mathrm{LP}_{(0)}$ is constituted as:

$\max \bar{Z}$

s.t. $\quad \sum_{\mathrm{i}=1}^{3} \sum_{\mathrm{j}=1}^{4} \mathrm{x}_{\mathrm{ij}} \leq 10^{10}$

$$
\begin{aligned}
7.087 \mathrm{x}_{11}-0.3304 \mathrm{x}_{12}+10.2261 \mathrm{x}_{13}-2.887 \mathrm{x}_{14}+3.3913 \mathrm{x}_{21}-5.1652 \mathrm{x}_{22}+0.8087 \mathrm{x}_{23} \\
+5.6696 \mathrm{x}_{24}+5.8087 \mathrm{x}_{31}+11.087 \mathrm{x}_{32}-1.3304 \mathrm{x}_{33}+2.3913 \mathrm{x}_{34}+5870 \bar{Z}=6650
\end{aligned}
$$

$\mathbf{x} \in S$.

The optimum solution of (8) is $\overline{\mathbf{x}^{*}}=(0,0,0,150,0,250,0,0,150,0,50,0)$.

Step 3: Since $1 . \bar{x}^{*} \neq 10^{10}$, then $\mathbf{x}^{1}=\overline{\mathbf{x}^{*}}$, and the numerator and denominator of the fractional objective are calculated as $Z^{1}=\frac{7000}{5370} \cong 1.3035$.

Step $4_{\text {iv }}$ : Since $Z^{0} \neq Z^{1}$, then put $k=1$, and return to Step 2 .

Step 2: The $\operatorname{LFTP}-\mathrm{LP}_{(1)}$ is constituted as: 
$\max \bar{Z}$

s.t. $\quad \sum_{\mathrm{i}=1}^{3} \sum_{\mathrm{j}=1}^{4} \mathrm{x}_{\mathrm{ij}} \leq 10^{10}$

$$
\begin{aligned}
9.7143 \mathrm{x}_{11} & +1.7714 \mathrm{x}_{12}+13.0286 \mathrm{x}_{13}-1.4857 \mathrm{x}_{14}+5.1429 \mathrm{x}_{21}-4.1143 \mathrm{x}_{22}+3.0857 \mathrm{x}_{23} \\
& +7.7714 \mathrm{x}_{24}+8.0857 \mathrm{x}_{31}+13.7143 \mathrm{x}_{32} 0.7714 \mathrm{x}_{33}+4.1429 \mathrm{x}_{34}+5370 \bar{Z}=7000
\end{aligned}
$$

$\mathbf{x} \in S$.

The optimum solution of (9) is $\overline{\mathbf{x}^{*}}=(0,0,0,150,0,250,0,0,150,0,50,0)$

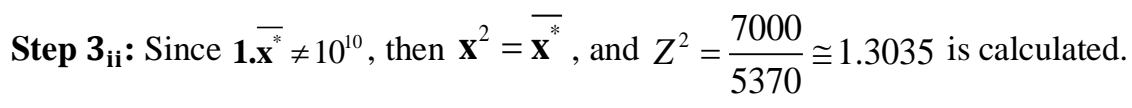

Step $\mathbf{4}_{\text {iii }}$ : Since $Z^{2}=Z^{1}$, then the algorithm ends with the optimum solution $\mathbf{x}^{*}=\mathbf{x}^{2^{*}}=(0,0,0,150,0,250,0,0,150,0,50,0)$ and the objective function value is $Z^{*}=\frac{7000}{5370} \cong 1.3035$.

We state that our algorithm finds the same optimum solution with the transportation simplex method (Bajalinov, 2003). However, our algorithm generates the optimum solution by applying two iterations containing one linear programming problem instead of many computations.

Example 2 (Gupta et al., 1993) (Asymptotic case): The following LFTP is considered

$$
\begin{array}{ll}
\min & Z(\mathbf{x})=\frac{5 x_{11}+4 x_{12}+2 x_{13}+6 x_{21}+5 x_{22}+3 x_{23}+8 x_{31}+9 x_{32}+4 x_{33}}{6 x_{11}+3 x_{12}+4 x_{13}+7 x_{21}+4 x_{22}+2 x_{23}+6 x_{31}+5 x_{32}+2 x_{33}} \\
\text { s.t. } & x_{11}+x_{12}+x_{13} \geq 5 \\
& x_{21}+x_{22}+x_{23} \geq 10 \\
& x_{31}+x_{32}+x_{33} \leq 9 \\
& x_{11}+x_{21}+x_{31} \geq 8 \\
& x_{12}+x_{22}+x_{32} \geq 15 \\
& x_{13}+x_{23}+x_{33} \leq 6 \\
& \mathbf{x} \geq 0
\end{array}
$$

Let $S$ denotes the feasible region.

Step 1: The initial solution $\mathbf{x}^{0}=(0,15,0,10,0,0,0,0,0)$ is determined, and the fractional objective $Z^{0}=\frac{120}{115} \cong 1.04348$ is achieved. We also choose $M=10^{10}$.

Step 2: Using $\mathbf{x}^{0}$ and $Z^{0}$, the problem LFTP-LP( $(0)$ is constituted as:

$\min \bar{Z}$

s.t. $\sum_{\mathrm{i}=1}^{3} \sum_{\mathrm{j}=1}^{3} \mathrm{x}_{\mathrm{ij}} \leq 10^{10}$

$$
\begin{aligned}
1.2609 \mathrm{x}_{11}-0.8695 \mathrm{x}_{12}+ & 2.1739 \mathrm{x}_{13}+1.3043 \mathrm{x}_{21}-0.8261 \mathrm{x}_{22}-0.913 \mathrm{x}_{23} \\
& -1.7391 \mathrm{x}_{31}-3.7826 \mathrm{x}_{32}-1.913 \mathrm{x}_{33}+115 \bar{Z}=120
\end{aligned}
$$

$$
\mathbf{x} \in S
$$


The optimum solution of (10) is $\overline{\mathbf{x}^{*}}=(0,0,5,8,11.9895,0,0,3.0105,0)$.

Step $\mathbf{3}_{\mathrm{i}}$ : Since $\mathbf{1 . \mathbf { x } ^ { * }} \neq 10^{10}$, then $\mathbf{x}^{1}=\overline{\mathbf{x}^{*}}$, and the fractional objective is calculated as $Z^{1}=\frac{145.042}{139.0105} \cong 1.04338$.

Step $4_{\text {iv }}$ : Since $Z^{0} \neq Z^{1}$, then put $k=1$, and return to Step 2 .

Step 2: The $\operatorname{LFTP}-\mathrm{LP}_{(1)}$ is constituted as:

$\min \bar{Z}$

s.t. $\sum_{\mathrm{i}=1}^{3} \sum_{\mathrm{j}=1}^{3} \mathrm{x}_{\mathrm{ij}} \leq 10^{10}$

$$
\begin{aligned}
1.2603 \mathrm{x}_{11}-0.8698 \mathrm{x}_{12}+ & 2.1736 \mathrm{x}_{13}+1.3037 \mathrm{x}_{21}-0.8264 \mathrm{x}_{22}-0.9132 \mathrm{x}_{23} \\
& -1.7397 \mathrm{x}_{31}-3.7831 \mathrm{x}_{32}-1.9132 \mathrm{x}_{33}+139.01 \bar{Z}=145.042
\end{aligned}
$$

$\mathbf{x} \in S$.

The optimum solution of (11) is $\overline{\mathbf{x}^{*}}=(0,0,6,10.6302,15,0,0,0,0)$.

Step 3: Since $1 \cdot \overline{\mathbf{x}^{*}} \neq 10^{10}$, then $\mathbf{x}^{2}=\overline{\mathbf{x}^{*}}$, and $Z^{2}=\frac{150.7812}{158.4114} \cong 0.9518$ is calculated.

Step $4_{\text {iv }}$ : Since $Z^{2} \neq Z^{1}$, then put $k=2$, and return to Step 2 .

Step 2: Using $\mathrm{x}^{2}$ and $Z^{2}$, the problem LFTP-LP(2) is constituted as:

$\min \bar{Z}$

s.t. $\sum_{\mathrm{i}=1}^{3} \sum_{\mathrm{j}=1}^{3} \mathrm{x}_{\mathrm{ij}} \leq 10^{10}$

$0.711 \mathrm{x}_{11}-1.1445 \mathrm{x}_{12}+1.8073 \mathrm{x}_{13}+0.6628 \mathrm{x}_{21}-1.1927 \mathrm{x}_{22}-1.0963 \mathrm{x}_{23}$

$\mathbf{x} \in S$.

$$
-2.289 \mathrm{x}_{31}-4.2408 \mathrm{x}_{32}-2.0963 \mathrm{x}_{33}+158.4114 \bar{Z}=150.7812
$$

The optimum solution of (12) is $\overline{\mathbf{x}^{*}}=(0,15,6,237.0321,0,0,0,0,0)$.

Step $3_{\mathrm{i}}$ : Since $1 . \overline{\mathbf{x}^{*}} \neq 10^{10}$, then $\mathbf{x}^{3}=\overline{\mathbf{x}^{*}}$, and the fractional objective is calculated as $Z^{3}=\frac{1494.1926}{1728.2247} \cong 0.8646$.

Step $4_{\text {iv }}$ : Since $Z^{3} \neq Z^{2}$, then put $k=3$, and return to Step 2 .

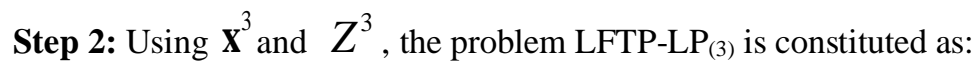

$\min \bar{Z}$

s.t. $\sum_{\mathrm{i}=1}^{3} \sum_{\mathrm{j}=1}^{3} \mathrm{x}_{\mathrm{ij}} \leq 10^{10}$

$$
\begin{aligned}
0.1875 \mathrm{x}_{11}-1.4062 \mathrm{x}_{12}+1 . & 4583 \mathrm{x}_{13}+0.0521 \mathrm{x}_{21}-1.5417 \mathrm{x}_{22}-1.2708 \mathrm{x}_{23} \\
& -2.8125 \mathrm{x}_{31}-4.6771 \mathrm{x}_{32}-2.2708 \mathrm{x}_{33}+1728.2247 \bar{Z}=1494.1926
\end{aligned}
$$

$\mathbf{x} \in S$.

The optimum solution of (13) is $\overline{\mathbf{x}^{*}}=(8032.0790,15,6,10,0,0,0,0,0)$. 
Step $3_{\mathrm{i}}$ : Since $1 . \overline{\mathbf{x}^{*}} \neq 10^{10}$, then $\mathbf{x}^{4}=\overline{\mathbf{x}^{*}}$, and the fractional objective is calculated as $Z^{4}=\frac{40292.395}{48331.474} \cong 0.83367$.

Step $4_{\text {iv }}$ : Since $Z^{4} \neq Z^{3}$, then put $k=4$, and return to Step 2 .

Step 2: The LFTP-LP(4) is constituted as:

$\min \bar{Z}$

s.t. $\sum_{\mathrm{i}=1}^{3} \sum_{\mathrm{j}=1}^{3} \mathrm{x}_{\mathrm{ij}} \leq 10^{10}$

$$
\begin{aligned}
0.002 \mathrm{x}_{11}-1.499 \mathrm{x}_{12}+1.3347 \mathrm{x}_{13}-0.1643 \mathrm{x}_{21}-1.6653 \mathrm{x}_{22}-1.3326 \mathrm{x}_{23} \\
-2.998 \mathrm{x}_{31}-4.8317 \mathrm{x}_{32}-2.3327 \mathrm{x}_{33}+48331.474 \bar{Z}=40292.395
\end{aligned}
$$

$\mathbf{x} \in S$.

The optimum solution of (14) is $\overline{\mathbf{x}^{*}}=(0,15,6,10,0,0,0,0,0)$.

Step 3: Since $1 . \mathbf{x}^{*} \neq 10^{10}$, then $\mathbf{x}^{5}=\overline{\mathbf{x}^{*}}$, and $Z^{5}=\frac{132}{139} \cong 0.9496$ is calculated.

Step $4_{\mathrm{ii}}: Z^{5}>Z^{4}$, so the limit value of the objective function at the asymptotic solution of the LFTP is $\frac{40292.395}{48331.474} \cong 0.83367$. Namely, $\inf _{x \in S} Z(\mathbf{x})=Z^{4}$.

Thus, the solution generated by our algorithm yields a better objective function value than Gupta et al.'s (1993) optimum solution. A comparison of the solutions is presented in Table 1.

Table 1: Comparison of the solutions for Example 2.

\begin{tabular}{ccc}
\hline & Gupta et al.'s Method & Proposed Method \\
\hline $\mathbf{X}^{*}$ & $(5,9,6,4,6,0,0,0,0)$ & $(8032.0790,15,6,10,0,0,0,0,0)$ \\
$\mathbf{Z}^{*}$ & $\frac{127}{133} \cong 0.9549$ & 0.83367 \\
& & \\
\hline
\end{tabular}

\subsection{Practical applications}

Let us consider a part of supply chain network of a company which produces and markets textile products. It is desired to implement logistic processes most efficiently by focusing on customer satisfaction. In the part of network, the products are transported from six distribution centers to meet ten customer zones demand. By minimizing the carbon footprint by taking into account the $\mathrm{CO}_{2}$ emission that occurs during transportation, an environmentalist perspective will be gained to the supply chain management. The requirements of the customer zones are respectively $4830,2900,4910,2720,4800,2760,3740,4520,7460,3500$ units of product and the supply of distribution centers are respectively $6600,9040,7800,9600,7200,1900$ units of product. Moreover, the data of profit and transportation cost for one unit of product to be transported from $i^{\text {th }}$ distribution center to $j^{\text {th }}$ customer zone are given in Table 2- 
3. How many units should be transported from each distribution centers to each customer zones in order that the profitability ratio of the company, expressed as profit/total $\mathrm{CO}_{2}$ emission, is maximum with the fixed amount of $\mathrm{CO}_{2}$ emission of processing at distribution centers being 165000 units?

Table 2: Profit of transportation from Distribution Centers (DC)s to Customer Zones (CZ)s.

\begin{tabular}{lllllll}
\hline & DC1 & DC2 & DC3 & DC4 & DC5 & DC6 \\
\hline CZ1 & 15.3625 & 11.1725 & 24.875 & 8.35 & 5.2725 & 2.9705 \\
CZ2 & 16.2935 & 23.69 & 0.375 & 9.5125 & 28.2925 & 3.0975 \\
CZ3 & 16.575 & 19.5725 & 15.625 & 9.4325 & 18.375 & 1.7875 \\
CZ4 & 13.025 & 31.125 & 12.375 & 11.25 & 22.0925 & 1.7935 \\
CZ5 & 17.325 & 21.475 & 21.95 & 16.275 & 23.175 & 7.875 \\
CZ6 & 17.015 & 16.55 & 8.35 & 22.625 & 15.625 & 6.625 \\
CZ7 & 7.795 & 7.175 & 8.125 & 22.175 & 4.135 & 13.375 \\
CZ8 & 6.995 & 7.45 & 6.745 & 12.25 & 5.905 & 5.1875 \\
CZ9 & 4.175 & 6.345 & 6.425 & 11.125 & 4.865 & 24.075 \\
CZ10 & 4.0625 & 3.225 & 3.55 & 9.125 & 3.075 & 20.375 \\
\hline
\end{tabular}

Table 3: Amount of $\mathrm{CO}_{2}$ emission of transportation from Distribution Centers (DC)s to Customer Zones (CZ)s.

\begin{tabular}{lllllll}
\hline & DC1 & DC2 & DC3 & DC4 & DC5 & DC6 \\
\hline CZ1 & 0.755625 & 6.0825 & 14.18875 & 14.7755 & 11.2725 & 31.9705 \\
CZ2 & 8.29775 & 7.52 & 58.775 & 15.95125 & 3.2925 & 32.0975 \\
CZ3 & 8.24575 & 3.95725 & 5.9625 & 11.4325 & 3.2375 & 28.89875 \\
CZ4 & 6.0925 & 1.39125 & 6.95375 & 11.3825 & 6.0925 & 28.79375 \\
CZ5 & 10.3125 & 5.9475 & 2.295 & 10.22375 & 4.9175 & 27.27875 \\
CZ6 & 9.015 & 8.73755 & 14.31875 & 8.65725 & 14.7625 & 23.3625 \\
CZ7 & 17.695 & 17.2875 & 18.9925 & 7.5375 & 23.135 & 10.63375 \\
CZ8 & 22.06895 & 19.4025 & 23.5745 & 12.625 & 25.905 & 12.41875 \\
CZ9 & 27.28275 & 23.3745 & 23.1425 & 12.7625 & 25.865 & 8.1175 \\
CZ10 & 26.0625 & 26.2225 & 26.2225 & 15.80125 & 28.89375 & 4.03775 \\
\hline
\end{tabular}

The fractional programming of the above transportation problem is as follows: 


$$
\begin{gathered}
\max Z(\mathbf{x})=\frac{\sum_{\mathrm{i}=1}^{6} \sum_{\mathrm{j}=1}^{10} \mathrm{p}_{\mathrm{ij}} \mathrm{x}_{\mathrm{ij}}}{\sum_{\mathrm{i}=1}^{6} \sum_{\mathrm{j}=1}^{10} \mathrm{c}_{\mathrm{ij}} \mathrm{x}_{\mathrm{ij}}+165000} \\
\text { subject to } \quad \sum_{\mathrm{j}=1}^{10} \mathrm{x}_{\mathrm{ij}} \leq \mathrm{a}_{\mathrm{i}}, \mathrm{i}=1, \ldots, 6 \\
\sum_{\mathrm{i}=1}^{6} \mathrm{x}_{\mathrm{ij}} \geq \mathrm{b}_{\mathrm{j}}, \mathrm{j}=1, \ldots, 10 \\
\mathrm{x}_{\mathrm{ij}} \geq 0, \forall \mathrm{i}, \mathrm{j}
\end{gathered}
$$

where $\mathbf{P}=\left[\mathrm{p}_{\mathrm{ij}}\right]_{6 \times 10}$ and $\mathbf{C}=\left[\mathrm{c}_{\mathrm{ij}}\right]_{6 \times 10}$ are matrices for profit and $\mathrm{CO}_{2}$ emission of transportation respectively, $\mathbf{a}=\left[\mathrm{a}_{\mathbf{i}}\right]=[6600,9040,7800,9600,7200,1900]$ is the vector of supply of the distribution centers, $\mathbf{b}=\left[\mathrm{b}_{\mathrm{j}}\right]=[4830,2900,4910,2720,4800,2760,3740,4520,7460,3500]$ is the vector of demand of the customer zones.

The model, which is equivalent to equation (6) given in the theory, was constituted with 17 constraints and 60 variables. All the computational experiments were performed by using the software GAMS 35.1.0 on a computer with a $2.7 \mathrm{GHz}$ processor and 8 GB RAM running Windows 10 . The optimum solution set $\mathbf{x}$ of the problem (15) is

$$
\mathbf{x}=\left[\mathrm{x}_{\mathrm{ij}}\right]_{6 \times 10}=\left[\begin{array}{cccccccccc}
0 & 0 & 0 & 0 & 0 & 2760 & 0 & 2240 & 0 & 1600 \\
0 & 0 & 4040 & 2720 & 0 & 0 & 0 & 2280 & 0 & 0 \\
4830 & 0 & 0 & 0 & 1370 & 0 & 0 & 0 & 1600 & 0 \\
0 & 0 & 0 & 0 & 0 & 0 & 3740 & 0 & 5860 & 0 \\
0 & 2900 & 870 & 0 & 3430 & 0 & 0 & 0 & 0 & 0 \\
0 & 0 & 0 & 0 & 0 & 0 & 0 & 0 & 0 & 1900
\end{array}\right]
$$

with a resulting the value of fractional objective with the nominator and denominator $\mathrm{Z}=\frac{77471.1}{593611}=1.305082$.

In addition to application, the large-scale transportation problems with fractional objective are constructed by generating data randomly. A company transports the units from supply nodes to demand points. Number of Supply Constraints (NoSC), Number of Demand Constraints (NoDC) and Number of Decision Variables (NoDV) indicates the size of generated problems. For each test problem, we generate all parameters as uniformly random numbers. We observe that the effect of NoSC, NoDC and NoDV on the total execution time and the iteration number of our method and present the results in Table 4.

We note that just the optimum solution case is taken into consideration to test our algorithm. For each class, ten test problem are generated randomly and solved with the software GAMS 35.1.0. The results indicates that our introduced procedure can overcome the optimum solution for different sizes of the LFTP problem within an acceptable time and number of iterations, even with large-scale problem. 
Table 4: Results of generated LFTP problems solving with the proposed algorithm.

\begin{tabular}{llllllll}
\hline Class & (NoSC, NoDC, NoDV) & \multicolumn{3}{c}{ Iterations } & \multicolumn{3}{c}{ Execution time } \\
\cline { 3 - 7 } & & Min & Max & Average & Min & Max & Average \\
\hline 1 & $(10,10,100)$ & 5 & 3.8 & 0.457 & 0.784 & 0.612 \\
2 & $(25,25,625)$ & 4 & 6 & 4.8 & 0.686 & 0.93 & 0.784 \\
3 & $(50,50,2500)$ & 5 & 6 & 5.6 & 0.785 & 1.689 & 1.018 \\
4 & $(75,75,5625)$ & 6 & 8 & 6.5 & 1.32 & 1.711 & 1.461 \\
5 & $(100,100,10000)$ & 6 & 8 & 6.8 & 1.829 & 2.631 & 2.13 \\
6 & $(200,200,40000)$ & 5 & 5 & 5 & 4.756 & 5.961 & 5.003 \\
7 & $(250,250,62500)$ & 4 & 5 & 4.9 & 6.457 & 8.682 & 7.649 \\
8 & $(500,500,250000)$ & 4 & 4 & 4 & 36.59 & 44.156 & 40.386 \\
9 & $(750,750,562500)$ & 4 & 4 & 4 & 155.546 & 132.53 & 147.443 \\
10 & $(1000,1000,1000000)$ & 3 & 4 & 3.5 & 364.021 & 434.986 & 328.149 \\
\hline
\end{tabular}

\section{Conclusion}

In this study, we expanded the iterative method developed by Ozkok (2020) for the LFP problem to LFTP. Firstly, it is considered that the objective function is continuous when linearization is performed for the proposed solution procedure based on the traditional definition of continuity. Then, a linear iterative structure is constructed with the linearization. This iterative method plays an important role in solving LFTP in real-life situations due to the effectiveness and easiness of computation. Our approach overcomes successfully the shortcoming in terms of computation by reason of increasing process as the problem size raises since it is an iterative procedure based on LP unlike existing methods. The proposed algorithm can be easily applied to even large-scale LFTPs since it solves LFTP by a series of LP problems. Another advantage of our algorithm is that it has the ability to solve the LFTP with mixed constraints since the method does not depend on constraints. Moreover, the case of asymptotic solution for LFTP was handled for the first time. The numerical examples and a case study were executed to demonstrate the proposed mathematical solution method, and the solutions of the examples were also compared with existing methods. Finally, the proposed method was performed on randomly generated large-scale problems, and computational results were presented. We coded the proposed algorithm in the software GAMS 35.1.0. For further study, the expanded iterative solution procedure can be adapted to different kinds of LFTP (i.e interval or solid LFTP) thanks to its flexibility of algorithm. As another future research, the algorithm can be improved to solve fuzzy LFTPs since introducing the parameters with fuzzy numbers would give more realistic result in modelling of real situations.

\section{Acknowledgment}

The authors would like to thank reviewers for their valuable suggestions and comments to improve the manuscript. 


\section{References}

1. Anukokila, P., Radhakrishnan, B., \& Anju, A. (2019). Goal programming approach for solving multiobjective fractional transportation problem with fuzzy parameters. RAIRO-Operations Research, 53(1), 157-178.

2. Bajalinov, E. B. (2003). Linear-fractional programming theory, methods, applications and software (Vol. 84). Springer Science \& Business Media.

3. Bitran, G. R., \& Novaes, A. G. (1973). Linear programming with a fractional objective function. Operations Research, 21(1), 22-29.

4. Cetin, N., \& Tiryaki, F. (2014). A fuzzy approach using generalized dinkelbach's algorithm for multiobjective linear fractional transportation problem. Mathematical Problems in Engineering, 2014.

5. Charnes, A., \& Cooper, W. W. (1962). Programming with linear fractional functionals. Naval Research logistics quarterly, 9(3-4), 181-186.

6. Gupta, A., Khanna, S., \& Puri, M. C. (1993). A paradox in linear fractional transportation problems with mixed constraints. Optimization, 27(4), 375-387.

7. Gupta, K., \& Arora, R. (2017). Solving the problem of industry by finding paradox in fractional plus fractional capacitated transportation problem. Advanced Modeling and Optimization, 19(2).

8. Gupta, S., Ali, I., \& Ahmed, A. (2018). Multi-choice multi-objective capacitated transportation problem-a case study of uncertain demand and supply. Journal of Statistics and Management Systems, 21(3), 467-491.

9. Güzel, N., Emiroglu, Y., Tapci, F., Guler, C., \& Syvry, M. (2012). A solution proposal to the interval fractional transportation problem.

10. Javaid, S., Jalil, S. A., \& Asim, Z. (2017). A model for uncertain multi-objective transportation problem with fractional objectives. Int J Oper Res, 14(1), 11-25.

11. Liu, S. T. (2016). Fractional transportation problem with fuzzy parameters. Soft computing, 20(9), 36293636.

12. Moanta, D. (2007). Some aspects on solving a linear fractional transportation problem. Journal of Applied Quantitative Methods, 2(3), 343-348.

13. Ozkok, B. A. (2020). An iterative algorithm to solve a linear fractional programming problem. Computers \& Industrial Engineering, 140, 106234.

14. Pradhan A., Biswal, M. P. (2015, December) Computational methodology for linear fractional transportation problem. In 2015 Winter Simulation Conference (WSC), 3158-3159 IEEE.

15. Raina, A. A., Gupta, S., \& Kour, K. (2018). Fractional Transportation Problem with non-linear discount cost. Sri Lankan Journal of Applied Statistics, 18, 3.

16. Sadia, S., Gupta, N., \& Ali, Q. M. (2016). Multiobjective capacitated fractional transportation problem with mixed constraints. Math Sci Lett, 5(3), 235-242.

17. Safi, M. R., \& Ghasemi, S. M. (2017). Uncertainty in linear fractional transportation problem. International Journal of Nonlinear Analysis and Applications, 8(1), 81-93.

18. Schaible, S. (1981). Fractional programming: applications and algorithms. European Journal of Operational Research, 7(2), 111-120.

19. Sheikhi, A., Karbassi, S. M., \& Bidabadi, N. (2018). A New Method for Solving Bi-Objective Fractional Transportation Problems.

20. Sivri, M., Emiroglu, I., Guler, C., \& Tasci, F. (2011, April). A solution proposal to the transportation problem with the linear fractional objective function. In 2011 Fourth International Conference on Modeling, Simulation and Applied Optimization (pp. 1-9). IEEE.

21. Swarup, K. (1966). Transportation technique in linear fractional functional programming. Journal of royal naval scientific service, 21(5), 256-260.

22. Tantawy, S. F. (2008). A new procedure for solving linear fractional programming problems. Mathematical and Computer Modelling, 48(5-6), 969-973. 